\title{
Lipid-Based Nanocarriers for RNA Delivery
}

\author{
Hui Yi Xue, Pengbo Guo", Wu-Cheng Wen ${ }^{\#}$ and Ho Lun Wong*
}

School of Pharmacy, Temple University, 3307 North Broad Street, Philadelphia, Pennsylvania, US 19140

\begin{abstract}
RNA-interference (RNAi) agents such as small-interfering RNA (siRNA) and micro-RNA (miRNA) have strong potential as therapeutic agents for the treatment of a broad range of diseases such as malignancies, infections, autoimmune diseases and neurological diseases that are associated with undesirable gene expression. In recent years, several clinical trials of RNAi therapeutics especially siRNAs have been conducted with limited success so far. For systemic administration of these poorly permeable and easily degradable macromolecules, it is obvious that a safe and efficient delivery platform is highly desirable. Because of high biocompatibility, biodegradability and solid track record for clinical use, nanocarriers made of lipids and/or phospholipids have been commonly employed to facilitate RNA delivery. In this article, the key features of the major sub-classes of lipid-based nanocarriers, e.g. liposomes, lipid nanoparticles and lipid nanoemulsions, will be reviewed. Focus of the discussion is on the various challenges researchers face when developing lipid-based RNA nanocarriers, such as the toxicity

Ho Lun Wong of cationic lipids and issues related to PEGylated lipids, as well as the strategies employed in tackling these challenges. It is hoped that by understanding more about the pros and cons of these most frequently used RNA delivery systems, the pharmaceutical scientists, biomedical researchers and clinicians will be more successful in overcoming some of the obstacles that currently limit the clinical translation of RNAi therapy.
\end{abstract}

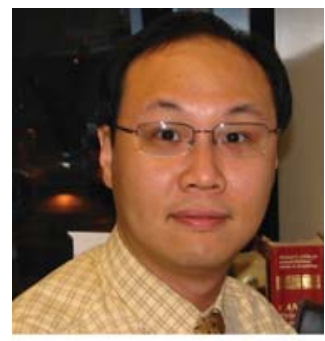

Keywords: Drug delivery, lipid, nanocarrier, RNA interference, small-interfering RNA.

\section{INTRODUCTION}

RNA-interference (RNAi) has been identified as a crucial biomolecular process to regulate gene expression. Small, non-coding RNA molecules were shown to mediate the RNAi effect in many ways, most notably by inducing mRNA degradation in cytoplasm as a part of RNA-induced silencing complex (RISC), which subsequently turns down the expression of the corresponding protein [1]. Since the report of RNAi effect in 1998 [2], different classes of RNAi agents, including small-interfering RNAs (siRNAs), microRNAs (miRNAs) and PIWI-interacting RNAs (piRNAs), have been discovered as useful RNAi mediators [3], and extensive progress has already been made to understand the biomolecular mechanism of RNAi. More recent studies also revealed other cellular activities such as immunostimulatory effects. For more details please refer to the updated reviews [3-5].

Considering the possibility of silencing the expression of almost any gene in a convenient, potent, and moderately specific manner, it is not surprising that researchers have been actively tapping into the application potential of RNAi. siRNAs have been extensively studied for biomedical applications as they are highly customizable. miRNAs also gather more and more attention as their deregulation have been identified as key causes of many pathological conditions $[5,6]$. Nowadays siRNAs and miRNAs have already become standard experimental tools to validate gene functions [6]. The prospect of therapeutic use of RNAi agents is even more appealing [7]. Researchers have studied the use of RNAi for management of diverse gene-related medical conditions including cancer, autoimmune diseases, neuropathological conditions and viral infections [7-12]. The potential of RNAi therapy is being supported by extensive in vitro and in vivo data $[7,13]$. Currently, the focus of RNAi research has shifted to clinical translation. Several trials, mostly on siRNAs, have been conducted and listed in Table $\mathbf{1}$.

\footnotetext{
*Address correspondence to this author at the School of Pharmacy, Temple University, 3307 North Broad Street, Philadelphia, Pennsylvania, US 19140; Tel: 215-707-8173; Fax: 215-707-3678; E-mail: ho-lun.wong@temple.edu or holunwong2011@yahoo.com

"P.G. and W-C.W. contributed equally to this work.
}

During these trials, a number of major issues that limit the success of RNAi therapy have been identified. One critical challenge is to achieve efficient and target-specific RNA delivery. RNA drugs are inherently difficult to be delivered especially by systemic route. Their high molecular weights (e.g. 13-14 kDa for a typical siRNA molecule) and poly-anionic nature limit their cell permeation and hence transfection efficiency. Naked RNA molecules are easily degraded so they are short-lived after systemically administered [7, $8,13]$. RNA drugs also lack target specificity which may increase the risk of triggering immunogenic responses $[14,15]$. Naked RNA are therefore more often used for local delivery [15]. For systemic delivery, a well-designed nanocarrier system will be valuable for solving at least some of the above issues. Non-viral carriers made of polymeric materials such as LODER polymer and cyclodextrinbased polymer have been employed in clinical trials [15], but as shown in Table 1, nanocarriers made of lipids and/or phospholipids, or lipid-based nanocarriers, have been more often used for RNA delivery in clinical setting because of advantages such as good biocompatibility, biodegradability, track record of biomedical uses (e.g. liposomes). Hence, it is time to take a focused look at lipidbased RNA nanocarriers. The goal of this review is to provide pharmaceutical scientists, biomedical researchers and clinicians a better understanding of this most important class of RNA delivery systems, including their designs, advantages, challenges and prospects for future development, in the hope for better success in the clinical translation of RNAi therapy.

\section{LIPID-BASED RNA NANOCARRIERS}

Because viral carriers are associated with a higher risk of triggering lethal immunogenic responses, non-viral nanocarriers have been the current choice for RNA delivery. Nanocarriers made of lipids and/or phospholipids are often used because of various favorable properties. To begin with, the cell membrane mainly consists of lipids and phospholipids. Lipid-based nanocarriers therefore have a natural tendency to interact well with the cell membrane to facilitate cellular uptake of RNA [16]. There are many choices of highly biocompatible and biodegradable lipids and phospholipids that are commercially available without the need for chemical synthesis. In addition, the risk of undesirable immunogenic reactions to 
Table 1. A list of clinical trials of RNA therapeutics systemically delivered.

\begin{tabular}{|c|c|c|c|c|c|}
\hline Clinical Trial Identifier & Year Started & Phase of trial & Disease & RNA & Carrier \\
\hline NCT01808638 & 2013 & $\mathrm{I} / \mathrm{II}$ & Pancreatic cancer & siRNA vs PKN3 & Liposome $^{b}$ \\
\hline NCT00927459 & 2009 & I & Hyper-cholesterolemia & siRNA vs ApoB & \multirow[t]{7}{*}{$\mathrm{LNP}^{\mathrm{c}}$} \\
\hline NCT01158079 & 2010 & I & Solid tumor & $\begin{array}{c}\text { siRNA vs VEGF and } \\
\text { KSP }\end{array}$ & \\
\hline NCT01148953 & 2010 & I & ATTR $^{d}$ & \multirow[t]{3}{*}{ siRNA vs TTR } & \\
\hline NCT01559077 & 2012 & I & ATTR & & \\
\hline NCT01617967 & 2012 & II & ATTR & & \\
\hline NCT01437007 & 2011 & I & Primary and secondary liver cancer & siRNA vs PLK1 & \\
\hline NCT01518881 & 2012 & I & Ebola virus infection & $\begin{array}{c}\text { siRNA vs ZEBOV L } \\
\text { polymerase, VP24 and } \\
\text { VP35 }\end{array}$ & \\
\hline NCT01858935 & 2013 & I & Fibrosis & siRNA vs HSP47 & $\begin{array}{l}\text { Vitamin A- coupled } \\
\text { lipid nanoparticle }\end{array}$ \\
\hline NCT01591356 & 2013 & I & Solid tumor & siRNA vs EphA2 & $\mathrm{DOPC}^{\mathrm{e}}$ \\
\hline
\end{tabular}

${ }^{a}$ Consists of cyclodextrin-containing polymer CAL101, stabilizing agent AD-PEG, and targeting agent AD-PEG-Tf for transferrin receptor, ${ }^{\mathrm{b}}$ Consists of cationic lipid AtuFECT01, neutral helper lipid DPhyPE, and PEGylated lipid DSPE-mPEG, ${ }^{\mathrm{c}}$ Lipid nanoparticles, ${ }^{\mathrm{d}}$ transthyretin mediated amyloidosis, ${ }^{\mathrm{e}}$ 1,2-dioleoyl-sn-glycero-3-phosphatidylcholine neutral liposome

lipids is also relatively lower than most of the polymeric materials which generally have much higher molecular weights.

Several classes of lipid-based nanocarriers have been studied for RNA delivery, the most notable ones being liposomes, lipidbased nanoparticles and lipid nanoemulsions. Lipid-based nanocarriers including other materials such as polymer, also known as hybrid nanocarriers, have also been studied and will be discussed later. Table 2 further summarizes their strengths and potential drawbacks for RNA delivery.

\section{Liposomes}

Liposomes are vesicles composed of a phospholipid bilayer with an aqueous core $[16,17]$. They can be easily prepared with biocompatible lipid/phospholipid ingredients, and can be conveniently tailored with functionalized lipids such as PEG-lipids for extended circulation and lipids conjugated with targeting moieties for tissue-specific delivery. With the presence of an aqueous compartment at the center, unlike other lipid-based systems which are almost exclusively used for loading lipophilic compounds, liposomes can be easily developed for the entrapment of hydrophilic and ionic drug molecules. Consequently, liposomes are probably the most commonly used nanosystems for delivery of RNAi agents. For years, cationic liposomes have been the standard carriers of RNA [17]. The positive charges of the lipids can improve the RNA encapsulation efficiency as the result of electrostatic interactions. Sometimes the process will result in spontaneous formation of siRNA/lipid complexes which are often referred as lipoplexes.
This complexation-based approach, however, has been challenged as some RNA molecules are still exposed on the carrier surface. A study showed that this could increase the risk of inducing immunogenic responses, and the IgM production could be reduced if the siRNA molecules were encapsulated instead of being complexed to the liposomes [18]. Researchers probably need to take his effect into consideration when designing liposomal RNA formulations. Liposomes made of cationic lipids are also quite toxic in general, but unfortunately negatively charged liposomes tend to have short in vivo circulation time due to quick clearance. This issue will be addressed in the later section.

\section{Nanoparticles}

When compared with liposomes, lipid-based nanoparticles such as solid lipid nanoparticles generally have solid, lipophilic core regions so they are inherently difficult to truly encapsulate the hydrophilic, poly-anionic RNA molecules. As a result, there are relatively few lipid nanoparticles for RNA delivery. However, this structure also opens up the possibility to load an RNA/small molecule drug combination by coating the RNA on the nanoparticle surface and encapsulating the lipophilic drugs in the core region [19]. For instance, Chen et al. developed a novel multifunctional nanoparticle composed of anionic lipids to deliver VEGF siRNA with doxorubicin [20]. The particle has high entrapment efficiency of both siRNA and the drug, building a strong base for the codelivery of the components. The results show an enhanced growth inhibition of the ovarian tumor cells. Also, the nanoparticles can also be used for the co-delivery of c-Myc siRNA and doxorubicin. 
Table 2. Summary of the strengths and potential limitations of lipid-based nanocarriers.

\begin{tabular}{|c|c|c|}
\hline Nanocarrier Type & Strengths & Potential limitations \\
\hline Liposomes & 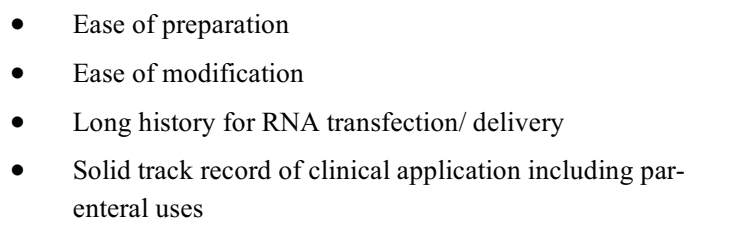 & $\begin{array}{l}\text { - } \\
\text { - } \\
\text { RNA may not be truly encapsulated in lipo- } \\
\text { plexes; reduced stability and increased risk } \\
\text { of immunogencity }\end{array}$ \\
\hline Lipid nanoparticles & $\begin{array}{ll}\text { - } & \text { Potential for controlled release } \\
\text { - } & \text { Physically stable } \\
\text { - } & \text { Preparation of solid lipid nanoparticles easily upscalable } \\
\text { - } & \text { May load one drug on surface and one drug in particle core }\end{array}$ & $\begin{array}{l}\text { - May not encapsulate RNA well in the solid } \\
\text { core } \\
\text { - No track record of clinical use of parenteral } \\
\text { lipid nanoparticle formulations }\end{array}$ \\
\hline Nanoemulsions & $\begin{array}{ll}\text { - } & \text { Ease of preparation } \\
\text { - } & \text { Ease to load multiple therapeutic agents }\end{array}$ & $\begin{array}{l}\text { - Liquid based formulations, may be less sta- } \\
\text { ble } \\
\text { - } \quad \text { Size of droplets may be too large }\end{array}$ \\
\hline
\end{tabular}

Because of the high biocompatibility of lipid-based nanocarriers with normal tissues, there are studies to explore the use of this class of RNA delivery platforms for non-cancer diseases. For example, Yamada et al. conducted an in vitro study of delivery of micro-RNA by liposome-based devices to pancreatic cells. They managed to load the 2'-OMe RNA onto the $\beta$-MEND nanoparticle and transfect the MIN6 cells [21]. The results showed high affinity between the nanoparticles and MIN6 cells and significant induction of insulin secretion. The research indicated the potential and promising application of the lipid based nanoparticles in treating diabetes.

\section{Lipid Nanoemulsions}

Nanoemulsions are thermodynamically stable isotropic systems in which two immiscible liquids (typically water and oil) are mixed to form a single phase, often with the help of appropriate surfactants/co-surfactants [22]. It was suggested that the oil phase may serve to protect the RNA from enzymatic degradation. For instance, Brito et al. [23] described a cationic nanoemulsion (CNE) for delivery of a self-amplifying RNA vaccine. The CNE/RNA complex was observed with good protection to the RNA under 16 hours of RNAase treatment. This RNA vaccine was able to elicit immune responses, especially cell-mediated responses, in mice, rats, rabbits and nonhuman primates with low doses. Overall, excellent biocompatibility was shown.

One advantage of nanoemulsions is the relative ease to mix multiple ingredients in a single formulation. For example, a radioopaque nanoemulsion for co-delivery of paclitaxel and Bcl-2 siRNA was studied [24]. An imaging contrasting agent was incorporated with paclitaxel as the hydrophobic oil core, whereas siRNA was electrostatically attached onto the PEGylated cationic outer shell. Flow cytometry data indicated that this nanoemulsion could effectively deliver the siRNA and paclitaxel into cells in vitro and the tumor tissue in vivo.

Up to date relatively few lipid nanoemulsions have been developed for RNA delivery because of a number of technical difficulties. As a liquid-based formulation it is difficult to immobilize the hydrophilic RNA molecules within the fine oil droplets. The RNA molecules will have a natural tendency to migrate to the water-oil interfaces and diffuse into the external medium with poor control. By convention, nanoemulsions are made of negatively charged ingredients so it is hard to stably entrap these RNA molecules. Another issue is about the size of the oil droplets. It is relatively diffi- cult to keep the size of droplets small and uniform during storage after inclusion of large molecules like RNA; processes such as coalescence and Ostwald ripening may occur to the droplets [22]. Cationic nanoemulsions have been studied for tackling these issues. Kaneda et al. [25] reported a nanoemulsion system that consists of a perfluorocarbon core covered with positively charged DOTAP for complexation with siRNA. Interestingly, although the size of nanoemulsion was approximately $300 \mathrm{~nm}$ in diameter, the nanoemulsion was able to efficiently deliver siRNA by the lipid raft mediated transport without trafficking to the Golgi or endoplasmic reticulum. The targeting of this alternative cellular uptake pathway by lipid nanoemulsion likely deserves more investigation in future.

\section{LIPID-BASED NANOCARRIERS FOR RNA DELIVERY - THE CHALLENGES}

Given the favorable properties of lipid-based nanocarriers for RNA delivery, there are a number of issues to address for this class of delivery systems to perform optimally at in vivo or clinical levels. Many issues contribute to the sub-optimal toxicity profiles of the supposedly safe lipid nanocarriers.

\section{Toxicity of Cationic Lipids}

As mentioned before, cationic lipids are typically included in lipid nanoformulations of RNAi therapeutics to improve RNA encapsulation and stability. Unfortunately, this class of lipid ingredients is also associated with significant toxicity issues [26]. A typical cationic lipid molecule is composed of three regions including a cationic head, a hydrophobic hydrocarbon backbone and a linker region [27]. A cationic lipid molecule can disrupt the integrity of a membrane structure as it resembles a detergent. At low concentration, a lipoplex consisting of cationic lipid molecules can irritate the exposed cell and cause cytoplasm vacuolization, reduced number of mitoses and cell shrinkage [28, 39]. When the lipoplex level is sufficiently high, cell lysis and necrosis may be triggered [30]. Cell toxicity may also be induced by interaction of the cationic groups with cellular enzymes such as protein kinase C [31]. Researchers also showed that at sub-lethal concentrations, cationic lipids can still alter the expression of multiple genes in undesirable manner. For instance, Oligofectamine was found to affect the expression of genes involved in cell apoptosis. Targets including caspase 8 isoform c, bcl-2-related protein a1, heat shock protein 70 , heat shock $60 \mathrm{kDa}$ protein 1 and Lipofectamine upregulated replication protein a1 can be up-regulated, and therefore the increasing risk of premature cell death by apoptosis [32]. 
At preclinical and clinical levels, systemic toxicities of lipoplexes have also been well-documented. Capture of lipoplexes by Kupffer cells in liver can trigger inflammatory responses such as release of tumor necrosis factor- $\alpha$, interferon- $\gamma$, interleukin- 6 and 12. These responses may inflict damages to liver and result in elevated levels in major liver function indicators such as AST and ALT. Leukopenia and thrombocytopenia may also occur [33]. At high dose of lipoplexes administered by systemic route, mortality of mice was also observed [34, 35].

There were studies suggesting that by using more labile, biodegradable linkers, the lipid molecules will degrade faster, leading to reduced toxicity [36]. However, this may potentially compromise the stability and transfection efficiency of the lipids, so careful optimization is required.

\section{Issues of PEGylation}

Lipid materials by nature interact well with cell surfaces in a non-specific fashion. These non-specific lipid-membrane interactions are even stronger in those lipid carriers of RNA as cationic lipids are often included and cell membranes are negatively charged. Cationic lipids-nucleic acid complexes administered in vivo were also reported to interact extensively with the negatively charged serum proteins and hence formation of aggregates and elimination by organs such as spleen, lung and liver [37]. Overall, the aforementioned properties will translate into short in vivo halflives after systemic administration. PEGylation (surface coating with polyethylene glycol groups) is therefore almost an indispensable standard practice nowadays when designing lipid nanocarriers of nucleic acids. However, PEGylation is not without its own drawbacks.

For effective RNAi-mediated gene silencing, the RNA loaded nanocarriers need to efficiently enter the target cells by endocytosis, and the RNA payload has to escape the endosomal compartment and enter the cytoplasm. The presence of hydrophilic PEG moieties on nanocarrier surfaces can reduce their interaction with cell membranes and consequently compromise the efficiency of these two crucial steps. In fact, this effect has been demonstrated not only in lipoplexes but also other non-lipid nanoparticles [38, 39].

RNAi therapeutics especially siRNAs are known for therapeutic activities that last for a few days up to a week. This warrants multiple dosing for chronic medical conditions. However, there are a number of studies reporting immunogenic responses elicited by PEGylated nanocarriers. It has been documented that empty PEGylated liposomes administered by systemic route can accelerate the clearance of the subsequently injected liposomes [40]. This is now known as "accelerated blood clearance $(\mathrm{ABC})$ phenomenon. This $\mathrm{ABC}$ phenomenon was later shown to be caused by production of anti-PEG IgM, and subsequent activation of complement system and accelerated capture of liposomes by Kupffer cells [41]. A study on multi-dosing of stabilized plasmid lipid particles also revealed loss of expression in the targeted tumor and increased expression in the liver in the later doses as a result of production of $\operatorname{IgM}$ and $\operatorname{IgG}$ against PEG [42].

\section{Other Issues}

Although lipids and phospholipids have superior biocompatibility and biodegradability compared with most biomaterials, it is inherently difficult to efficiently and stably incorporate the hydrophilic, poly-anionic RNA molecules into a lipophilic environment. The common practice is to use cationic lipids as previously discussed, but this increases the toxicity. Reducing the cationic charges on the nanocarriers, however, not only may reduce the RNA encapsulation efficiency, but also the transfection efficiency and subsequently the therapeutic effectiveness. This issue is particularly a concern for lipid nanoemulsions, which are mostly made of neutral and anionic lipids in liquid state and have difficulty in immobilizing the cationic lipids to avoid causing excessive toxicity [22]. How to achieve a balance of acceptable toxicity and good performance remains a key challenge in developing lipid-based RNA nanocarriers.

Conventional lipoplexes are based on ionic complexation between the lipid and RNA molecules of opposite charges. In other words, the RNA molecules are not truly encapsulated within the nanocarrier systems. Not only this may increase risk of immunogenic responses as previously mentioned [18], this also implies a lack of good control over how, when and where the RNA molecules leave the delivery system. Overall, it is important to develop more advanced nanocarrier systems with the RNA therapeutics encapsulated within the carrier cores and tighter control over the RNA release kinetics.

\section{STRATEGIES TO OVERCOME THE CHALLENGES}

To keep the strengths of lipid-based nanocarriers of RNA, and overcome the various issues associated with these delivery systems, several strategies have been explored in recent years. Table 3 summarizes these strategies. The details are being discussed as follows.

\section{Lipid Modification}

As the cationic lipids are the key source of the toxicity of lipidbased RNA nanocarriers, there have been many attempts to discover or synthesize less toxic cationic lipids. Proper modifications and engineering of the lipid molecule structure can also improve the transfection performance. There are many strategies developed for cationic lipid engineering for improved nucleic acid delivery and readers can refer to the specialized reviews for details [43, 44]. In brief, a phospholipid molecule typically consists of a cationic head, a hydrophobic hydrocarbon backbone and a linker region, and researchers can selectively alter the structure of one or more of the three regions for improved delivery and transfection performance. A classic example is DOTAP. Its linker consists of biodegradable ester bonds so the toxic effects of the lipids become relatively shortterm and moderate $[45,46]$. DOTAP thus has become the gold standard for RNA transfection and for the more newly developed cationic lipids to compare with. Another example is the modification of the hydrophobic domain. Strategies such as including different alkyl chains in the same lipid, using asymmetrical backbone, optimization of alkyl chain length, and using steroid hydrophobic domain have been studied to enhance the transfection efficiency without increasing the toxicity. It should be noted that most of the documented information about the lipid toxicity is based on in vitro assays. There is also a general lack in the study of their immunogenicity, so one should exercise caution in choosing the "less toxic" cationic lipids for in vivo or clinical purpose.

\section{Use of Non-Cationic Lipids}

Although there are RNA nanocarriers that use only non-ionic or anionic lipids, in general this approach may compromise the RNA encapsulation, stability and transfection performance. Instead of totally replacing the cationic lipids, one possible strategy to reduce the toxicity is to combine non-cationic lipids with cationic lipids in nanocarrier preparation. In fact, neutral lipids are often included to improve various properties of the RNA nanocarriers. The so called fusogenic lipids such as 1-alpha dioleoyl phosphatidyl ethanolamine are often added to promote endosomal escape and cholesterol or other high melting lipids can be used to improve the physical stability of the nanocarriers $[47,48]$. Unfortunately, "diluting" the cationic lipids with neutral or anionic lipids does not always mitigate the toxicity. In some cases, the apparently less harmful lipids may actually potentiate the overall toxicity of the nanocarriers [49]. An example is the use of combination of DOTAP and cholesterol led to stronger non-specific cell killing effects than the DOTAP only nanosystem. Hence, lipid combinations need to be carefully evaluated if the main purpose is to achieve a less toxic nanocarrier. 
Table 3. Common strategies used to improve the performance of lipid nanocarriers of RNA.

\begin{tabular}{|c|c|c|}
\hline Strategy & Description & Comments \\
\hline Lipid modification & $\begin{array}{l}\text { Chemically engineer cationic lipids to achieve } \\
\text { less toxic lipids }\end{array}$ & $\begin{array}{l}\text { - Toxicity often screened by in vitro assays; data should serve } \\
\text { as guidelines only when applied clinically }\end{array}$ \\
\hline Use of non-cationic lipids & $\begin{array}{l}\text { Replacement some or all cationic lipids with } \\
\text { neutral or anionic lipids }\end{array}$ & $\begin{array}{l}\text { - Some neutral lipids can improve transfection, e.g. fusogenic } \\
\text { lipids to improve endosomal escape } \\
\text { - Sometimes non-cationic lipids can potentiate the toxicity of } \\
\text { the cationic components }\end{array}$ \\
\hline $\begin{array}{l}\text { High potency RNA nano- } \\
\text { formulations }\end{array}$ & $\begin{array}{l}\text { Use combinatorial synthesis and screening to } \\
\text { develop lipid-based nanoformulations with } \\
\text { improved RNAi potency }\end{array}$ & $\begin{array}{l}\text { - Lower dose of RNA can be used, which may reduce the risk of } \\
\text { toxicity and immunogenicity }\end{array}$ \\
\hline Hybrid nanocarriers & $\begin{array}{l}\text { Incorporate polymer into lipid carriers to } \\
\text { achieve better RNA encapsulation and im- } \\
\text { proved RNA release kinetics }\end{array}$ & $\begin{array}{l}\text { - The lipid components tend to reduce the toxicity of the po- } \\
\text { lymeric components }\end{array}$ \\
\hline $\begin{array}{l}\text { Stabilized RNA lipid } \\
\text { nanoparticle }\end{array}$ & $\begin{array}{l}\text { Optimized choice and ratio of various neutral, } \\
\text { cationic and PEGylated lipids/ phospholipids } \\
\text { to build a nanocarrier that is stable, efficient } \\
\text { and less toxic }\end{array}$ & $\begin{array}{l}\text { - The most successful type of nanocarriers so far used for RNA } \\
\text { delivery in terms of clinical translation } \\
\text { - Still carries risk of immunogenic responses }\end{array}$ \\
\hline Active-targeting & $\begin{array}{l}\text { Surface functionalized nanocarriers with re- } \\
\text { ceptor substrates, antibodies or cell- } \\
\text { penetrating peptides to increase specificity for } \\
\text { target cells }\end{array}$ & $\begin{array}{l}\text { - Has become the standard practice in developing RNA nano- } \\
\text { carriers nowadays } \\
\text { - May improve the RNA transfection efficiency by triggering } \\
\text { endocytosis }\end{array}$ \\
\hline
\end{tabular}

\section{High Potency RNA Nanoformulations}

Using combinatorial synthesis and screening of lipid-like (also known as lipidoids) materials, researchers successfully prepare nanoformulations that could knock down gene expression at very low siRNA dose levels $(<0.01 \mathrm{mg}$ and $0.03 \mathrm{mg}$ siRNA per $\mathrm{kg}$ body weight in mice and non-human primates, respectively) [50, 51]. This implies that a lower dose of nanocarriers is needed to achieve the same RNAi effect, so the RNA/nanocarrier toxicity can be effectively reduced.

In other studies, the potency of siRNA was substantially improved by substituting DOTAP with a newly developed cationic lipid DLinMC3DMA in the lipid nanoparticles. The $\mathrm{IC}_{50}$ was reduced from about $10 \mathrm{mg}$ siRNA/ $\mathrm{kg}$ body weight to $0.005 \mathrm{mg}$ siRNA/kg body weight with this change [52]. The authors suggested that the improvement is contributed by the use of ionizable cationic lipids with optimized $\mathrm{pKa}$ values and maximized endosomal bilayer destabilizing capabilities.

\section{Integrating Lipid and Polymeric Materials - Hybrid Nanocarri- ers}

Lipid-based nanomaterials are adept at controlling the drug release rates and have good biocompatibility and low immunogenicity [53], whereas polymeric materials tend to have good affinities with large molecules like RNA. Hence, there have been a number of "hybrid" nanosystems developed that combine lipids and polymers in order to improve encapsulation and delivery of RNA $[54,55]$. For example, hybrid nanoparticles were shown to reduce both the acute (necrotic and apoptotic) and longer term (proliferation suppression) cellular toxicities on non-cancer breast cells and prostate cells when compared to non-lipidated polyethylenimine while achieving high cell transfection efficiencies $[55,56]$. In another study, liposomes containing polymers, termed lipopolyplexes for carrying RNA were also shown to reduce the undesirable toxicity [57]. In another study, Ewe et al. have recently developed lipopolyplexes carrying siRNA and DNA and efficient delivery by inhaled route was demonstrated in vivo [58].

\section{Stabilized RNA Lipid Nanoparticle}

In terms of clinical development, LNP (lipid nanoparticle, in earlier reports also known as SNALP, stabilized nucleic acid lipid particle) is probably the most successful RNA nanocarrier studied up to date [59]. Many clinical trials of LNP for a range of diseases have been conducted (Table 1). In essence, this system has integrated many of the aforementioned strategies to address multiple issues associated with RNA delivery, including limited in vivo circulation, inefficient transfection, and unacceptable toxicity. A LNP, typically $120 \mathrm{~nm}$ in diameter or smaller, is prepared using a spontaneous vesicle formation method. The nanocarrier is composed of a combination of neutral lipids (e.g. cholesterol and 1,2-distearoylsn-glycero-3-phosphocholine), cationic lipids, and PEG-conjugated lipids [60]. Interestingly, a recent study indicated that LNP has a solid core, electron-dense complex structure with physico-chemical properties that totally different from liposomes, even it containing ingredients that are often seen in liposomes. [61]. It was suggested that by careful optimization of the choice and ratio of the various ingredients, LNP can achieve extended circulation, effective endosomal escape rate without triggering excessive production of cytokines.

The performance of LNPs in vivo and in clinical settings has been extensively evaluated. In animals, it was shown that there was no significant complement activation, pro-inflammatory cytokine production, delayed coagulation, or other changes in haematology parameters [62]. In a Phase I clinical trial evaluating LNP delivering siRNA against apolipoprotein B, no signs of hepatotoxicity were detected. Unfortunately, flu-like symptoms likely associated with immunostimulation by the high siRNA payload was observed in one of the subjects treated at the highest dose level. The manufacturer (Tekmira) therefore decided to terminate the trial early [63]. In brief, LNPs demonstrate excellent potential for RNA deliv- 
Table 4. Examples of targeting moieties used for active targeting by lipid nanocarriers of RNA.

\begin{tabular}{|c|c|c|}
\hline Class of targeting moieties & Example & References \\
\hline \multirow[t]{2}{*}{ Receptor substrate } & Folate & {$[55]$} \\
\hline & Apolipoprotein E & {$[65]$} \\
\hline \multirow[t]{2}{*}{ Antibody or its fragment } & anti-EGFR ${ }^{\mathrm{a}}$ antibody & [66] \\
\hline & Fab' fragments of anti-HB-EGF ${ }^{b}$ antibody & {$[67]$} \\
\hline \multirow[t]{2}{*}{ Peptide } & protamine-derived $\mathrm{CPP}^{\mathrm{c}}$ & [68] \\
\hline & lipopeptides from human papillomavirus type-16 capsid & {$[69]$} \\
\hline
\end{tabular}

${ }^{\mathrm{a}}$ EGFR - epidermal growth factor receptor; ${ }^{\mathrm{b}}$ anti-HB-EGF - anti-heparin-binding epidermal growth factor-like growth factor; ${ }^{\mathrm{c}} \mathrm{CPP}-\mathrm{cell}$ penetrating peptide

ery, but more toxicity data, especially related to the immunogenicity, are still needed to validate their clinical value [61].

\section{Active-Targeting}

By improving the target specificity, it is possible to lower the dose of an RNA nanoformulation so the toxicity issue will be addressed at that same time. Although the passive targeting strategy known as the enhanced permeation and retention (i.e. EPR) effect is able to increase the tumor specificity and tumor accumulation by RNA nanocarriers [64], it is only useful for cancer treatment and the improvement gained is often just modest. The more recently developed RNA nanocarriers therefore are typically surfacemodified with active-targeting moieties. Table 4 lists some examples of the moieties used for active targeting by lipid nanocarriers of RNA. These moieties include receptor substrates, monoclonal antibodies and cell-penetrating peptides [55, 65-69]. The surface modifications are technically not difficult for lipid-based systems as the phospholipid molecules especially those PEGylated ones can be easily functionalized to provide useful sites for conjugation to the targeting moieties. In fact, functionalized PEGylated lipids already conjugated with folate or transferrin are commercially available at affordable pricing. In addition to improving the delivery specificity, targeting of cell surface receptors may often trigger endocytosis, thus improving the RNA transfection efficiency as well. For example, Xue et al. compared the uptake of folate-decorated lipid nanoparticles by PC3 prostate cancer cells with non-targeted nanoparticles [55]. The flow cytometry data indicated an increase in transfection efficiency from around $20 \%$ to over $80 \%$. In short, this feature is now almost a standard in the development of RNA nanoformulations.

\section{CONCLUSION}

After over a decade since the discovery of the RNAi phenomenon, now is the time to move siRNAs and miRNAs to the clinical stage. At this point, the delivery issues and the related toxicity problems have been the biggest obstacles so far. Comparing lipid-based nanocarriers with synthetic polymer [e.g., polyethyleneimine, poly cationic polymers such as poly(L-lysine) and poly(L-histidine)]based carriers, lipid-based nanocarriers are apparently the most biocompatible class of delivery platforms that may help overcome this critical obstacle. Liposomes have a long track for parenteral use. From the clinical trials it is also clear that the immunogenic responses were mainly derived from the RNA drugs rather than the lipids. With high biocompatibility and low immunogenicity, the current trend is to further optimize the lipid-based RNA delivery system(to achieve more optimal RNA delivery systems) by integrating several of the strategies discussed in this review.

Meanwhile, researchers should still continue to develop the new generation of RNA therapeutics to reduce their immunogenicity, improve their stability and increase their potency. By combining safer RNA drugs with more efficient nanocarrier platforms, there is still a bright future for the clinical use of this class of therapeutics that can be easily synthesized and customized for a broad range of gene-related diseases.

\section{CONFLICT OF INTEREST}

The authors confirm that this article content has no conflicts of interest.

\section{ACKNOWLEDGEMENTS}

This work was supported by National Institutes of Health/National Cancer Institute R01 grant (R01CA168917).

\section{REFERENCES}

[1] Kumar LD, Clarke AR. Gene manipulation through the use of small interfering RNA (siRNA): From in vitro to in vivo applications. Adv Drug Deliv Rev 2007; 59(2): 87-100.

[2] Fire A, Xu S, Montgomery MK, Kostas SA, Driver SE, Mello CC. Potent and specific genetic interference by double-stranded RNA in Caenorhabditis elegans. Nature 1998; 391(6669): 806-11.

[3] Dogini DB, Pascoal VD, Avansini SH, Vieira AS, Pereira TC, Lopes-Cendes I. The new world of RNAs. Genet Mol Biol 2014; 37(1): 285-93.

[4] Ipsaro JJ, Joshua-Tor L. From guide to target: molecular insights into eukaryotic RNA-interference machinery. Nat Struct Mol Biol 2015; 22(1): 20-8.

[5] Mansoori B, Shotorbani SS, Baradaran B. RNA Interference and its Role in cancer therapy. Adv Pharm Bull 2014; 4(4): 313-21.

[6] Kumar LD, Clarke AR. Gene manipulation through the use of small interfering RNA (siRNA): From in vitro to in vivo applications. Adv Drug Deliv Rev 2007; 59(2): 87-100.

[7] Musacchio T, Torchilin VP. siRNA delivery: from basics to therapeutic applications. Front In Biosci 2012; 18: 58-79.

[8] Seth S, Johns R, Templin MV. Delivery and biodistribution of siRNA for cancer therapy: challenges and future prospects. Ther Deliv 2012; 3(2): 245-61.

[9] Oh Y-K, Park TG. siRNA delivery systems for cancer treatment. Adv Drug Deliv Rev 2009; 61(10): 850-62.

[10] Kaur IP, Sharma G. siRNA: a new approach to target neuropathic pain. Biodrugs 2012; 26(6): 401-12.

[11] Huda R, Tüzün E, Christadoss P. Complement C2 siRNA mediated therapy of myasthenia gravis in mice. J Autoimmun 2013; 42: 94104.

[12] Novobrantseva TI, Borodovsky A, Wong J, et al. Systemic RNAimediated gene silencing in nonhuman primate and rodent myeloid cells. Mol Ther Nucl Acids 2012; 1(1): e4.

[13] Gavrilov K, Saltzman WM. Therapeutic siRNA: principles, challenges, and strategies. Yale J Biol Med 2012; 85(2): 187.

[14] Ma Z, Li J, He F, Wilson A, Pitt B, Li S. Cationic lipids enhance siRNA-mediated interferon response in mice. Biochem Biophys Res Commun 2005; 330(3): 755-9.

[15] Draz MS, Fang BA, Zhang P, et al. Nanoparticle-mediated systemic delivery of siRNA for treatment of cancers and viral infections. Theranostics 2014; 4(9): 872-92. 
[16] Pathak K, Keshri L, Shah M. Lipid nanocarriers: influence of lipids on product development and pharmacokinetics. Crit Rev Ther Drug Carrier Syst 2011; 28(4): 357-93.

[17] Mallick S, Choi JS. Liposomes: versatile and biocompatible nanovesicles for efficient biomolecules delivery. J Nanosci Nanotechnol 2014; 14(1): 755-65.

[18] Tagami T, Uehara Y, Moriyoshi N, Ishida T, Kiwada H. Anti-PEG IgM production by siRNA encapsulated in a PEGylated lipid nanocarrier is dependent on the sequence of the siRNA. J Control Release 2011; 151(2): 149-54.

[19] Bae KH, Lee JY, Lee SH, Park TG, Nam YS. Optically traceable solid lipid nanoparticles loaded with siRNA and paclitaxel for synergistic chemotherapy with in situ imaging. Adv Healthcare Mater 2013; 2(4): 576-84.

[20] Chen Y, Bathula SR, Li J, Huang L. Multifunctional nanoparticles delivering small interfering RNA and doxorubicin overcome drug resistance in cancer. J Biol Chem 2010; 285(29): 22639-50.

[21] Yamada Y, Tabata M, Yasuzaki Y, et al. A nanocarrier system for the delivery of nucleic acids targeted to a pancreatic beta cell line. Biomaterials 2014; 35(24): 6430-8.

[22] Souto E, Nayak A, Murthy R. Lipid nanoemulsions for anti-cancer drug therapy. Pharmazie Int J Pharm Sci 2011; 66(7): 473-8.

[23] Brito LA, Chan M, Shaw CA, et al. A cationic nanoemulsion for the delivery of next-generation RNA vaccines. Mol Ther 2014; 22(12): 2118-29.

[24] Oh MH, Kim JS, Lee JY, Park TG, Nam YS. Radio-opaque theranostic nanoemulsions with synergistic anti-cancer activity of paclitaxel and Bcl-2 siRNA. RSC Adv 2013; 3(34): 14642-51.

[25] Kaneda MM, Sasaki Y, Lanza GM, Milbrandt J, Wickline SA. Mechanisms of nucleotide trafficking during siRNA delivery to endothelial cells using perfluorocarbon nanoemulsions. Biomaterials 2010; 31(11): 3079-86.

[26] Xue HY, Liu S, Wong HL. Nanotoxicity: a key obstacle to clinical translation of siRNA-based nanomedicine. Nanomedicine 2014; 9(2): 295-312.

[27] Simberg D, Weisman S, Talmon Y, Barenholz Y. DOTAP (and other cationic lipids): chemistry, biophysics, and transfection. Crit Rev Ther Drug Carrier Syst 2004; 21(4): 62.

[28] Lappalainen K, Jääskeläinen I, Syrjänen K, Urtti A, Syrjänen S. Comparison of cell proliferation and toxicity assays using two cationic liposomes. Pharm Res 1994; 11(8): 1127-31.

[29] Stoddart MJ. Cell viability assays: introduction. In: Stoddart MJ, Ed. Mammalian Cell Viability. USA: Humana Press 2011; pp. 1-6.

[30] Wu J, Lizarzaburu ME, Kurth MJ, et al. Cationic lipid polymerization as a novel approach for constructing new DNA delivery agents. Bioconjug Chem 2001; 12(2): 251-7.

[31] Aberle AM, Tablin F, Zhu J, Walker NJ, Gruenert DC, Nantz MH. A novel tetraester construct that reduces cationic lipid-associated cytotoxicity. Implications for the onset of cytotoxicity. Biochemistry $1998 ; 37(18): 6533-40$.

[32] Hollins AJ, Omidi Y, Benter IF, Akhtar S. Toxicogenomics of drug delivery systems: Exploiting delivery system-induced changes in target gene expression to enhance siRNA activity. J Drug Target 2007; 15(1): 83-8.

[33] Zhang J-S, Liu F, Huang L. Implications of pharmacokinetic behavior of lipoplex for its inflammatory toxicity. Adv Drug Deliv Rev 2005; 57(5): 689-98.

[34] Tousignant JD, Gates AL, Ingram LA, et al. Comprehensive analysis of the acute toxicities induced by systemic administration of cationic lipid: plasmid DNA complexes in mice. Hum Gene Ther 2000; 11(18): 2493-513.

[35] Tan Y, Huang L. Overcoming the inflammatory toxicity of cationic gene vectors. J Drug Target 2002; 10(2): 153-60.

[36] Choi JS, Lee EJ, Jang HS, et al. New cationic liposomes for gene transfer into mammalian cells with high efficiency and low toxicity. Bioconjug Chem 2001; 12(1): 108-13.

[37] Simões S, Filipe A, Faneca H, et al. Cationic liposomes for gene delivery. Expert Opin Drug Deliv 2005; 2(2): 237-54.

[38] Song LY, Ahkong QF, Rong Q, et al. Characterization of the inhibitory effect of PEG-lipid conjugates on the intracellular delivery of plasmid and antisense DNA mediated by cationic lipid liposomes. Biochim Biophys Acta Biomembr 2002; 1558(1): 1-13.

[39] Mishra S, Webster P, Davis ME. PEGylation significantly affects cellular uptake and intracellular trafficking of non-viral gene delivery particles. Eur J Cell Biol 2004; 83(3): 97-111.
[40] Dams ETM, Laverman P, Oyen WJG, et al. Accelerated blood clearance and altered biodistribution of repeated injections of sterically stabilized liposomes. J Pharmacol Exp Ther 2000; 292(3): 1071-9.

[41] Ishida T, Ichihara M, Wang XY, et al. Injection of PEGylated liposomes in rats elicits PEG-specific IgM, which is responsible for rapid elimination of a second dose of PEGylated liposomes. J Control Release 2006; 112(1): 15-25.

[42] Judge A, McClintock K, Phelps JR, et al. Hypersensitivity and loss of disease site targeting caused by antibody responses to PEGylated liposomes. Mol Ther 2006; 13(2): 328-37.

[43] Lv H, Zhang S, Wang B, et al. Toxicity of cationic lipids and cationic polymers in gene delivery. J Control Release 2006; 114(1): 100-9.

[44] Zhi DF, Zhang SB, Wang B, et al. Transfection efficiency of cationic lipids with different hydrophobic domains in gene delivery. Bioconjug Chem 2010; 21(4): 563-77.

[45] Swami A, Goyal R, Tripathi SK, et al. Effect of homobifunctional crosslinkers on nucleic acids delivery ability of PEI nanoparticles. Int J Pharm 2009; 374(1): 125-38.

[46] Breunig M, Lungwitz U, Liebl R, et al. Breaking up the correlation between efficacy and toxicity for nonviral gene delivery. Proc Natl Acad Sci USA 2007; 104(36): 14454-59.

[47] Simberg D, Weisman S, Talmon Y, et al. DOTAP (and other cationic lipids): chemistry, biophysics, and transfection. Crit Rev Therap Drug Carrier Syst 2004; 21(4): 257-317.

[48] Haque ME, McIntosh TJ, Lentz BR. Influence of lipid composition on physical properties and peg-mediated fusion of curved and uncurved model membrane vesicles: "Nature's own" fusogenic lipid bilayer. Biochemistry 2001; 40(14): 4340-8.

[49] Nchinda G, Überla K, Zschörnig O. Characterization of cationic lipid DNA transfection complexes differing in susceptability to serum inhibition. BMC Biotech 2002; 2(1): 12.

[50] Akinc A, Goldberg M, Qin J, et al. Development of lipidoidsiRNA formulations for systemic delivery to the liver. Mol Ther 2009; 17(5): 872-9.

[51] Love KT, Mahon KP, Levins CG, et al. Lipid-like materials for low-dose, in vivo gene silencing. Proc Natl Acad Sci USA 2010; 107(5): 1864-69.

[52] Jayaraman M, Ansell SM, Mui BL, et al. Maximizing the potency of siRNA lipid nanoparticles for hepatic gene silencing in vivo. Angew Chem Int Ed 2012; 51(34): 8529-33.

[53] Narvekar M, Xue HY, Eoh JY, et al. Nanocarrier for Poorly WaterSoluble Anticancer Drugs-Barriers of Translation and Solutions. AAPS PharmSciTech 2014; 1-12.

[54] Xue HY, Wong HL. Tailoring nanostructured solid-lipid carriers for time-controlled intracellular siRNA kinetics to sustain RNAimediated chemosensitization. Biomater 2011;32(10): 2662-72.

[55] Xue HY, Wong HL. Solid lipid-PEI hybrid nanocarrier: an integrated approach to provide extended, targeted, and safer siRNA therapy of prostate cancer in an all-in-one manner. ACS Nano 2011; 5(9): 7034-47.

[56] Xue HY, Narvikar M, Zhao JB, et al. Lipid encapsulation of cationic polymers in hybrid nanocarriers reduces their non-specific toxicity to breast epithelial cells. Pharm Res 2013; 30(2): 572-83.

[57] Masotti A, Moretti F, Mancini F, et al. Physicochemical and biological study of selected hydrophobic polyethylenimine-based polycationic liposomes and their complexes with DNA. Bioorg Med Chem 2007; 15(3): 1504-15.

[58] Ewe A, Aigne A. Nebulization of liposome-polyethylenimine complexes (lipopolyplexes) for DNA or siRNA delivery: Physicochemical properties and biological activity. Eur J Lipid Sci Technol 2014; 116: 1195-204.

[59] Rossi JJ. RNAi therapeutics: SNALPing siRNAs in vivo. Gene Ther 2005; 13(7): 583-84.

[60] Jeffs LB, Palmer LR, Ambegia EG, et al. A scalable, extrusion-free method for efficient liposomal encapsulation of plasmid DNA. Pharm Res 2005; 22(3): 362-72.

[61] Barros SA, Gollob JA. Safety profile of RNAi nanomedicines. Adv Drug Deliv Rev 2012; 64(15): 1730-7.

[62] Zimmermann TS, Lee ACH, Akinc A, et al. RNAi-mediated gene silencing in non-human primates. Nature 2006; 441(7089): 111-4.

[63] Tekmira Pharmaceuticals. Tekmira Pharmaceuticals completes ApoB SNALP Phase 1 clinical trial. [Posted 2010 Jan 7, Accessed 2013 Jun 20]. Available from: (http: //investor.tekmirapharm.com/releasedetail.cfm?releaseid=534353) 
[64] Greish K. Enhanced permeability and retention (EPR) effect for anticancer nanomedicine drug targeting. Cancer Nanotechnol 2010; 624: 25-37.

[65] Tamaru M, Akita H, Nakatani T, et al. Application of apolipoprotein E-modified liposomal nanoparticles as a carrier for delivering DNA and nucleic acid in the brain. Int J Nanomed 2014; 9: 4267.

[66] Mokhtarieh AA, Cheong S, Kim S, et al. Asymmetric liposome particles with highly efficient encapsulation of siRNA and without nonspecific cell penetration suitable for target-specific delivery. Biochim Biophys Acta Biomembr 2012; 1818(7): 1633-41.

Received: March 25, 2015
[67]

Okamoto A, Asai $\mathrm{T}$, Kato $\mathrm{H}$, et al. Antibody-modified lipid nanoparticles for selective delivery of siRNA to tumors expressing membrane-anchored form of HB-EGF. Biochem Biophys Res Commun 2014; 449(4): 460-5.

[68] Asai T, Tsuzuku T, Takahashi S, et al. Cell-penetrating peptideconjugated lipid nanoparticles for siRNA delivery. Biochem Biophys Res Commun 2014; 444(4): 599-604.

[69] Weyland M, Griveau A, Bejaud J, et al. Lipid nanocapsule functionalization by lipopeptides derived from human papillomavirus type-16 capsid for nucleic acid delivery into cancer cells. Int J Pharm 2013; 454(2): 756-64. 